\title{
Variability in Estimated Glomerular Filtration Rate by Area under the Curve Predicts Renal Outcomes in Chronic Kidney Disease
}

\author{
Szu-Chia Chen, ${ }^{1,2,3}$ Ming-Yen Lin, ${ }^{1}$ Teng-Hui Huang, ${ }^{4}$ Chi-Chih Hung, ${ }^{1,3}$ Yi-Wen Chiu, ${ }^{1,5}$ \\ Jer-Ming Chang, ${ }^{1,2,3}$ Jer-Chia Tsai, ${ }^{1,5}$ Shang-Jyh Hwang, ${ }^{1,5}$ and Hung-Chun Chen ${ }^{1,5}$ \\ ${ }^{1}$ Division of Nephrology, Department of Internal Medicine, Kaohsiung Medical University Hospital, Kaohsiung Medical University, \\ 100 Shih-Chuan 1st Road, Kaohsiung 807, Taiwan \\ ${ }^{2}$ Department of Internal Medicine, Kaohsiung Municipal Hsiao-Kang Hospital, Kaohsiung Medical University, Kaohsiung 807, Taiwan \\ ${ }^{3}$ Faculty of Medicine, College of Medicine, Kaohsiung Medical University, Kaohsiung 807, Taiwan \\ ${ }^{4}$ Department of Public Health, Kaohsiung Medical University, Kaohsiung 807, Taiwan \\ ${ }^{5}$ Faculty of Renal Care, College of Medicine, Kaohsiung Medical University, Kaohsiung 807, Taiwan
}

Correspondence should be addressed to Jer-Chia Tsai; jerchia@kmu.edu.tw

Received 16 July 2014; Revised 9 September 2014; Accepted 9 September 2014; Published 23 October 2014

Academic Editor: Allen R. Nissenson

Copyright (C) 2014 Szu-Chia Chen et al. This is an open access article distributed under the Creative Commons Attribution License, which permits unrestricted use, distribution, and reproduction in any medium, provided the original work is properly cited.

\begin{abstract}
Greater variability in renal function is associated with mortality in patients with chronic kidney disease (CKD). However, few studies have demonstrated the predictive value of renal function variability in relation to renal outcomes. This study investigates the predictive ability of different methods of determining estimated glomerular filtration rate (eGFR) variability for progression to renal replacement therapy (RRT) in CKD patients. This was a prospective observational study, which enrolled 1,862 CKD patients. The renal end point was defined as commencement of RRT. The variability in eGFR was measured by the area under the eGFR curve (AUC)\%. A significant improvement in model prediction was based on the -2 log likelihood ratio statistic. During a median 28.7-month follow-up, there were 564 (30.3\%) patients receiving RRT. In an adjusted Cox model, a smaller initial eGFR AUC\%_12M $(P<0.001)$, a smaller peak eGFR AUC\%_12M $(P<0.001)$, and a larger negative eGFR slope_12M $(P<0.001)$ were associated with a higher risk of renal end point. Two calculated formulas: initial eGFR AUC\%_12M and eGFR slope_12M were the best predictors. Our results demonstrate that the greater eGFR variability by AUC\% is associated with the higher risk of progression to RRT.
\end{abstract}

\section{Introduction}

Chronic kidney disease (CKD) is an increasing worldwide public health problem associated with increased morbidity and mortality $[1,2]$. Greater variability in renal function is associated with high mortality in CKD [3]. The mechanisms of variability in renal function are multifactorial, including intrinsic renal disease (e.g., renal microvascular disease or impaired autoregulatory mechanisms) and extrinsic factors (e.g., volume status and presence and severity of concurrent illness). However, only limited studies have demonstrated the predictive value of renal function variability in relation to renal outcomes in CKD patients.

Plotting slopes of $1 /$ creatinine or estimated glomerular filtration rate (eGFR) versus time has been used as a tool for outcome variable in clinical studies [4]. eGFR slope has been identified as an independent risk factor for cardiovascular morbidity and mortality and progression to end-stage renal disease [5-10]. It is necessary to obtain the summarized information in the multivariate data by repeated measurement. Dynamic fluctuations in eGFR slope change may contribute to additional prognostic information beyond cross-sectional data. The area under the curve (AUC) computed with the trapezoidal formula is widely used as an approach to measure the dynamic and accumulating change of clinical laboratory parameters [11-14], but little is known about its application in the repeated measurements of eGFR variability. The eGFR AUC\% formula by repeated measurements of eGFR was used as a measure of variability in renal function in our study. We 


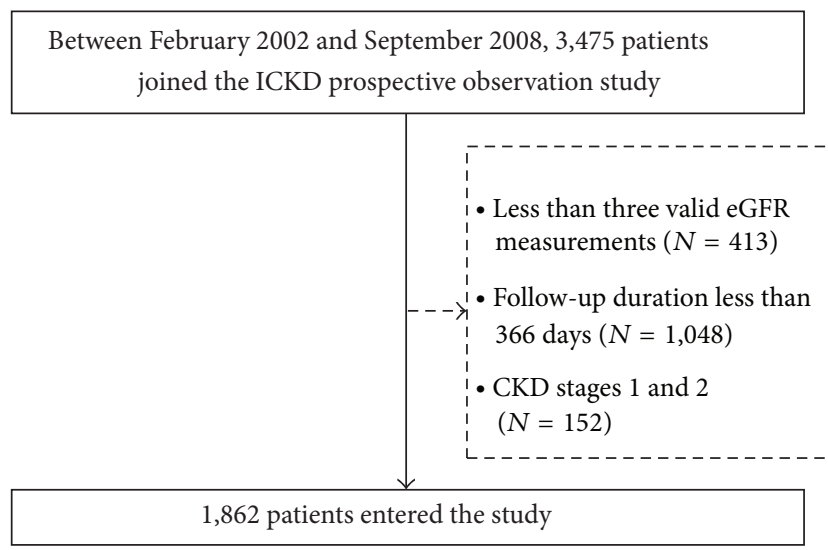

FIGURE 1: Derivation of patient cohort from all patients registered between February 2002 and September 2007; 1,862 patients were included in the analysis cohort.

hypothesize that eGFR AUC\%, a method of estimating renal function variability, is a useful predictor for renal outcomes in CKD patients. Hence, the purposes of the present study are (1) to determine whether eGFR AUC\% is associated with renal outcomes in progression to renal replacement therapy (RRT) and (2) to compare the predictive ability of renal outcomes using different methods of estimating renal function variability and eGFR slope in CKD stage 3-5 patients.

\section{Subjects and Methods}

2.1. Participants and Measurements. Between February 2002 and September 2008, 3,475 patients who joined the ICKD (Integrated CKD care program) prospective observation study from two affiliated hospitals (Kaohsiung Medical University Hospital and Kaohsiung Municipal Hsiao-Kang Hospital) of Kaohsiung Medical University were included and followed until May 2010. CKD was defined by using the National Kidney Foundation-Kidney Disease Outcomes Quality Initiative (K/DOQI) guidelines, and the CKD stage was classified using each subject's baseline eGFR [15]. Patients who had only two serum creatinine measurements during follow-up ( $n=413)$ or whose follow-up period was less than 12 months $(n=1048)$ were excluded. Besides, patients with CKD stages 1 and $2(n=152)$ were excluded. The final study population consisted of 1,862 CKD patients. Figure 1 showed the flowchart of the derivation of the cohort. Baseline variables included demographic features (age and sex), medical history (diabetes mellitus [DM], hypertension, and cardiovascular disease), examination findings (body mass index [BMI] and blood pressure), and laboratory data (albumin, fasting glucose, triglyceride, total cholesterol, hemoglobin, total calcium, phosphate, calcium-phosphorous product [Ca $\times \mathrm{P}$ product], uric acid, and urine protein-tocreatinine ratio). DM and hypertension were defined by clinical diagnosis. Cardiovascular disease was defined as clinical diagnosis of heart failure, acute or chronic ischemic heart disease, and cerebrovascular disease. The laboratory data 3
TABLE 1: Different methods to estimate variability of eGFR.

\begin{tabular}{ll}
\hline Method & Definition of calculation \\
\hline (A) Initial eGFR & $\begin{array}{l}\text { Initial eGFR is baseline value and estimated } \\
\text { variability of eGFR during 12 months (gray } \\
\text { area was divided into rectangle area) }\end{array}$ \\
\hline (B) Peak eGFR & $\begin{array}{l}\text { Peak eGFR is baseline value and estimated } \\
\text { variability of eGFR during 12 months (gray } \\
\text { area was divided into rectangle area) }\end{array}$ \\
\hline
\end{tabular}

eGFR: estimated glomerular filtration rate; AUC: area under the curve.

months before and after enrollment in the CKD care system were averaged and analyzed. In addition, information of medications using angiotensin converting enzyme inhibitors (ACEI) and angiotensin II receptor blockers (ARB) during the study period was obtained from medical records.

2.2. Quantification of Renal Function. Kidney function was quantified by using eGFR derived from the simplified Modification of Diet in Renal Disease (MDRD) Study equation. The equation was eGFR $\mathrm{mL} / \mathrm{min} / 1.73 \mathrm{~m}^{2}=186 \times$ Serum creatinine $e^{-1.154} \times$ Age $^{-0.203} \times 0.742$ (if female) [16] Serum creatinine was measured by the compensated Jaffé (kinetic alkaline picrate) method in a Roche/Integra 400 Analyzer (Roche Diagnostics, Mannheim, Germany) using a calibrator traceable to isotope-dilution mass spectrometry [17].

2.3. Definition of Renal End Point. The renal end point was defined as commencement of RRT. In patients reaching renal end point, renal function data were censored at the start of RRT. The other patients were followed until May 2010. The commencement of dialysis was determined according to the regulations by the National Health Service for dialysis therapy based on laboratory data, nutrition status, and uremic symptoms and signs.

2.4. Assessment of Rate of Renal Function Decline and Variability. The rate of renal function decline was assessed by the slope of eGFR, defined as the regression coefficient between eGFR and time in units of $\mathrm{mL} / \mathrm{min} / 1.73 \mathrm{~m}^{2} /$ year. At least three eGFR measurements were required to estimate eGFR slope. Faster renal function progression was reflected in a larger negative value of the slope. In addition, eGFR AUC\% was used to estimate the variability in eGFR. Table 1 showed the specifics of the calculations and illustrates two different methods of estimating eGFR AUC\%. A smaller eGFR AUC\% indicates greater eGFR variability. Initial eGFR AUC\%_12M was defined as initial eGFR for baseline value and estimated variability in eGFR during 12 months. Peak eGFR AUC\%_12M was defined as peak eGFR for baseline value and estimated variability in eGFR during 12 months. Figure 2 represents two cases to illustrate the eGFR variability.

2.5. Statistical Analysis. Statistical analysis was performed using SPSS version 17.0 (SPSS Inc., Chicago, IL, USA) for Windows. Data are expressed as percentages, mean \pm 


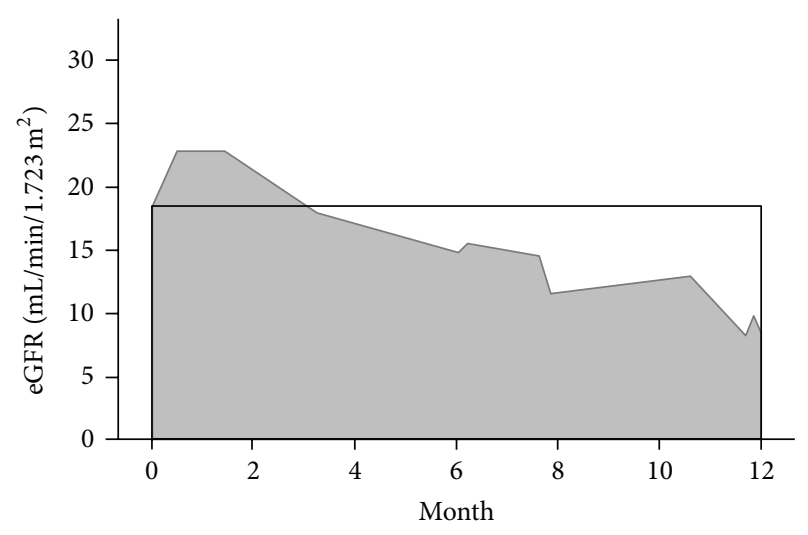

(a) Initial eGFR AUC\%_12M

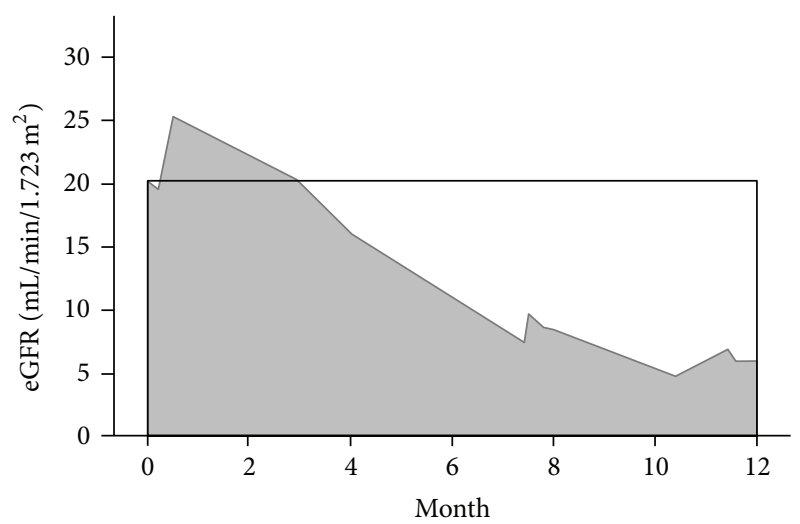

(c) Initial eGFR AUC\%_12M

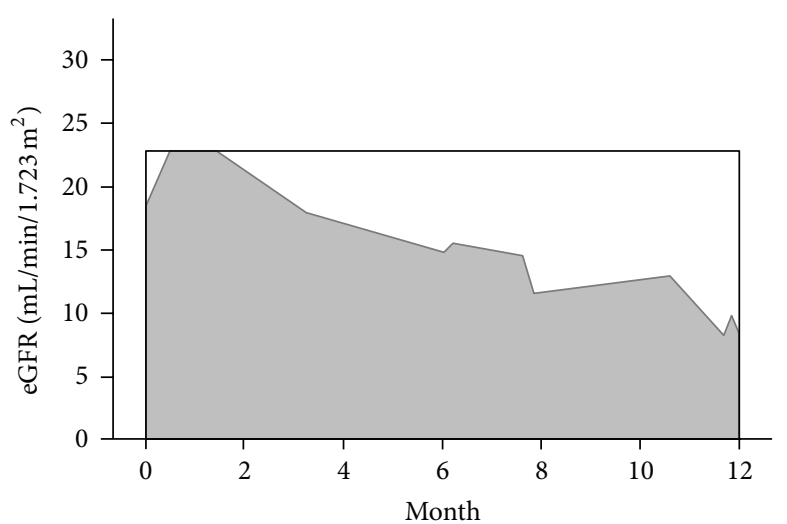

(b) Peak eGFR AUC\%_12M

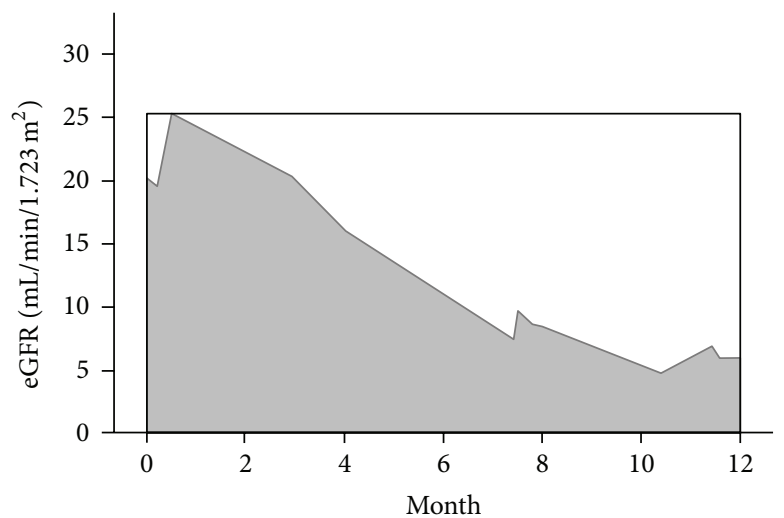

(d) Peak eGFR AUC\%_12M

FIGURE 2: Two representative cases to illustrate the eGFR variability. In Case 1, the initial eGFR AUC\%_12M (a) and peak eGFR AUC\%_12M (b) were $85.0 \%$ and $68.9 \%$, respectively. The eGFR slope of Case 1 was $-1.06 \mathrm{~mL} / \mathrm{min} / 1.73 \mathrm{~m}^{2}$ per month. In Case 2 , the initial eGFR AUC\%_12M (c) and peak eGFR AUC\%_12M (d) were $64.8 \%$ and $51.3 \%$, respectively. The eGFR slope of Case 2 was $-1.51 \mathrm{~mL} / \mathrm{min} / 1.73 \mathrm{~m}{ }^{2}$ per month. Case 2 had greater eGFR variability than that of Case 1.

standard deviation, or median (25th-75th percentile) for triglyceride, urine protein-to-creatinine ratio, and days of follow-up. The number of all missing baseline data was less than $2 \%$ except the data of ACEI and/or ARB use. This adjustment of ACEI and/or ARB use was accomplished using multiple imputation approach. The differences between groups were checked by chi-square test for categorical variables, by independent $t$-test for continuous variables with approximately normal distribution, or by Mann-Whitney $U$ test for continuous variables with skewed distribution. Cox proportional hazards analyses were used to investigate the relationships between different methods of estimating eGFR variability and eGFR slope with renal end point. The adjusted covariates included age, sex, a history of diabetes, hypertension, and cardiovascular disease, systolic and diastolic blood pressure, BMI, albumin, fasting glucose, triglyceride, total cholesterol, hemoglobin, eGFR, total calcium, phosphorous, CaXP product, uric acid, urine protein-to-creatinine ratio, ACEI and/or ARB use, and acute kidney injury episode. A significant improvement in model prediction was based on the $-2 \log$ likelihood ratio statistic, which followed a difference in likelihood ratio. The $P$ value was based on the incremental value compared with the basic model.
Differences were considered significant if the $P$ value was less than 0.05 .

\section{Results}

A total of 1,862 nondialyzed CKD patients were included. The mean age was $63.6 \pm 13.4$ years and there were 1,084 males and 778 females. The underlying etiologies of CKD in our patients included 646 with diabetic kidney disease (34.7\%), 647 with nondiabetic glomerular diseases (34.7\%), 200 cases of hypertension (10.7\%), and 369 caused by other diseases (19.8\%). The comparison of baseline characteristics between patients with and without renal end point was shown in Table 2. Compared with patients without renal end point, patients with renal end point were found to have a younger age, more female subjects, higher prevalence of DM and hypertension, higher systolic blood pressure, lower BMI, higher prevalence of advanced CKD stage, lower albumin, lower hemoglobin, lower baseline eGFR, lower calcium, higher phosphate, higher CaXP product, higher uric acid, higher urine protein-to-creatinine ratio, and higher prevalence of ACEI and/or ARB use. Besides, patients with renal end point had lower initial eGFR AUC\%_12M, lower 


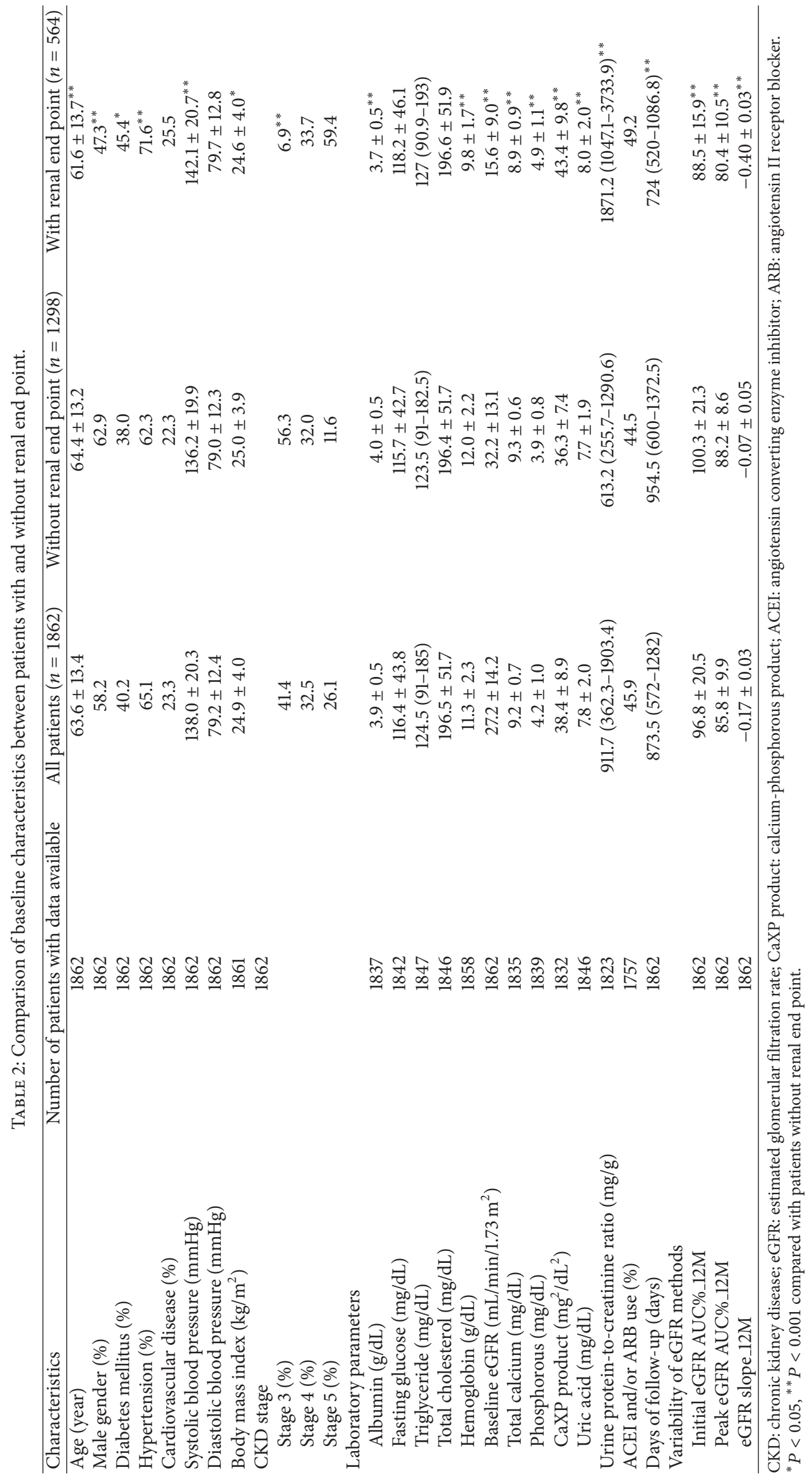


peak eGFR AUC\%_12M, and lower eGFR slope_12M. The study patients were stratified into two groups according to median values of initial eGFR AUC\%_12M (97.5\%) and peak eGFR AUC\%_12M (88.2\%). The comparison of clinical characteristics among study groups was shown in Table 3.

\subsection{Relation of eGFR Variability and eGFR Slope to Renal} End Points. The mean follow-up period was $28.7 \pm 14.0$ months. During the period of follow-up, five hundred and sixty-four patients (30.3\%) who started RRT were recorded in these 1,862 patients. Table 4 showed the hazard ratios (HR) of different methods of estimating eGFR variability and eGFR slope for renal end point, with adjustments for age, sex, a history of diabetes, hypertension, and cardiovascular disease, systolic and diastolic blood pressure, BMI, albumin, fasting glucose, triglyceride, total cholesterol, hemoglobin, eGFR, total calcium, phosphorous, $\mathrm{Ca} \times \mathrm{P}$ product, uric acid, urine protein-to-creatinine ratio, ACEI and/or ARB use, and acute kidney injury episode. Patients with small initial eGFR AUC\%_12M (HR, 0.962, 95\% CI, 0.955 to 0.969; $P<0.001$ ), small peak eGFR AUC\%_12M (HR, 0.973, 95\% CI, 0.966 to $0.980 ; P<0.001)$, and negative eGFR slope_12M (HR, $0.358,95 \% \mathrm{CI}, 0.309$ to $0.414 ; P<0.001$ ) were significantly associated with progression to renal end point. To clarify the effect of competitive risks between death and end-stage renal disease (ESRD), we further performed the multivariate analysis. We found that initial eGFR AUC\%_12M (HR, 0.961, 95\% CI, 0.954 to 0.968 ; $P<0.001$ ), peak eGFR AUC\%_12M (HR, 0.974, 95\% CI, 0.967 to 0.981; $P<0.001$ ), and eGFR slope_12M (HR, $0.279,95 \% \mathrm{CI}, 0.234$ to $0.332 ; P<0.001)$ were still significantly associated with progression to renal end point.

3.2. Incremental Values in eGFR Variability and eGFR Slope for Renal End Points. The incremental values in eGFR variability and eGFR slope, when they were added to the basic model to predict renal end point, were shown in Table 5. The addition of initial eGFR AUC\%_12M $(P<0.001)$, peak eGFR AUC\%_12M $(P<0.001)$, and eGFR slope_12M $(P<0.001)$ to the basic model significantly improved the predictive value of renal end points. The maximum change in the -2 log likelihood ratio was observed for initial eGFR AUC\%_12M, followed by eGFR slope_12M, and peak eGFR AUC\%_12M.

Besides, with regard to using standard error (SE) of the regression as a method of valuating eGFR variability, we found that the SE of the regression was not significantly associated with renal outcomes in multivariate analysis (HR, 0.926 ; $95 \% \mathrm{CI}, 0.699$ to $1.226 ; P=0.590)$.

\section{Discussion}

In the present study, we evaluated the association between eGFR variability and progression to RRT and compared the predictive ability of renal outcomes using different methods to assess eGFR variability in CKD patients. We found that smaller eGFR AUC\% or larger negative eGFR slope during 12 month follow-up periods was associated with a higher risk of progression to RRT and provided additional predictive value. Two calculated formulas initial eGFR AUC\%_12M and eGFR slope_12M were the best predictors.

The first important finding of our study is the identification of greater eGFR variability as a risk factor for adverse renal outcomes in CKD. Previous studies have identified eGFR slope as an independent risk factor for cardiovascular morbidity and mortality [5-10]. Al-Aly et al. [3] evaluated the impact of variability in renal function on mortality in a large sample size of CKD patients $(n=51,304)$. They used the coefficient of variation of the regression line coefficient fitted to all outpatient measures of eGFR to define variability in renal function and found that patients in the highest tertile of eGFR variability had an increased risk of death, independent of both baseline level of eGFR and eGFR slope. In addition, such variability provided further prognostic information beyond baseline eGFR and eGFR slope [3]. The reason for higher mortality in patients with greater variability in eGFR might be related to factors both intrinsic and extrinsic to the kidney and unmeasured exposures that may threaten renal homeostasis (e.g., changes in intravascular volume status, congestive heart failure, liver or pulmonary disease, or medications). In addition, hospital-acquired and frequent episodes of acute kidney injury, diuretics use, and cardiorenal syndrome all contribute to greater eGFR variability. Our present study revealed that greater eGFR variability, expressed as smaller eGFR AUC\%, was associated with progression to RRT and provided additional predictive value. This implies that variability in renal function is a potentially more important consideration in deciding which patients will progress to RRT.

The second important finding of our study is that one calculated formula initial eGFR AUC\%_12M was a better predictor of adverse renal outcomes than eGFR slope. Unlike other summary measures (e.g., baseline, average, or maximum eGFR value), both pieces of information can be captured through the use of AUC [18]. The use of AUC simplifies the statistical analyses by transforming the multivariate data into a univariate variable, especially when many repeated measurement numbers exist and there is a need to summarize the information [14]. In addition, when the time interval between repeated measurements is not identical, the use of AUC provides an alternative approach to adjust for these differences [19]. O'Hare et al. investigated the variability in eGFR in the 2 years before initiation of dialysis in 5,606 Veterans Affairs patients and found that patients with greater eGFR variability were less likely to have received predialysis care and had a higher risk of death in the first year after dialysis [20]. An understanding of kidney function variability preceding dialysis can help guide clinical decision making (e.g., nephrology referral, vascular assessment, and transplant referral), goals of care, and anticipated service needs [20-22]. The formula for eGFR AUC\% may also be used in long term follow-up in patients with CKD.

As for other approaches, such as using SE of the regression as a method of evaluating eGFR variability, our result is in agreement with Perkins RM's finding that eGFR variability by the SE did not predict ESRD outcome among CKD stage 3 patients [23]. Despite this finding, it needs further 


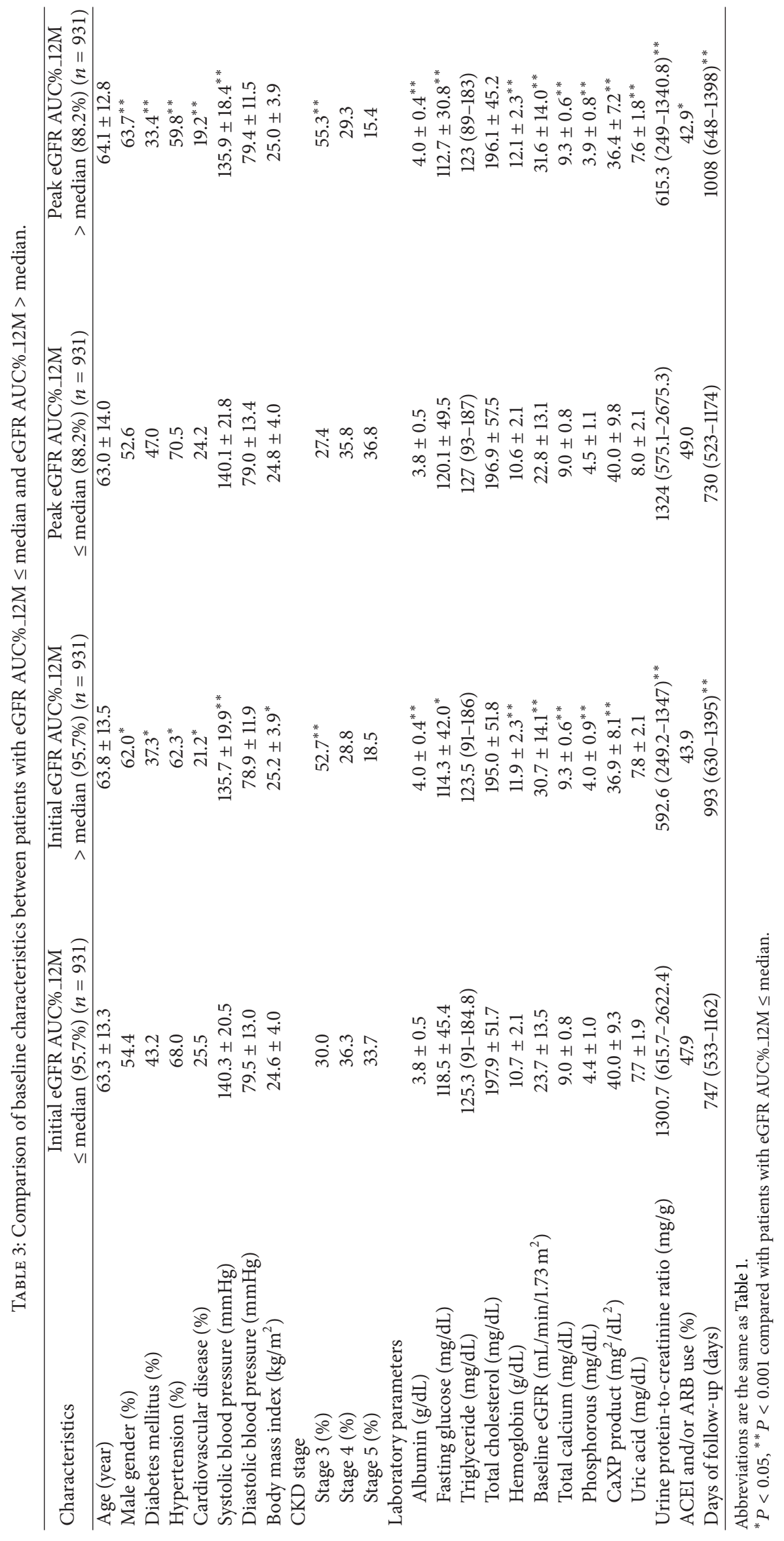


TABLE 4: Risk association between eGFR variability and renal end points using Cox proportion hazard model analysis.

\begin{tabular}{lcc}
\hline Method & HR $(95 \% \mathrm{CI})$ & $P$ value \\
\hline Initial eGFR AUC\%_12M & $0.962(0.955-0.969)$ & $<0.001$ \\
Peak eGFR AUC\%_12M & $0.973(0.966-0.980)$ & $<0.001$ \\
eGFR slope_12M & $0.358(0.309-0.414)$ & $<0.001$ \\
\hline
\end{tabular}

Values expressed as hazard ratio (HR) and $95 \%$ confidence interval (CI). Covariates in the multivariate model included age, sex, a history of diabetes, hypertension, and cardiovascular disease, systolic and diastolic blood pressure, body mass index, albumin, fasting glucose, triglyceride, total cholesterol, hemoglobin, eGFR, total calcium, phosphorous, CaXP product, uric acid, urine protein-to-creatinine ratio, ACEI and/or ARB use, and acute kidney injury episode.

TABLE 5: Predictive values of eGFR variability in relation to renal end points.

\begin{tabular}{lcc}
\hline Method & $\Delta-2 \log$ likelihood ratio & $P$ value \\
\hline Initial eGFR AUC\%_12M & 141.782 & $<0.001$ \\
Peak eGFR AUC\%_12M & 42.258 & $<0.001$ \\
eGFR slope_12M & 136.870 & $<0.001$ \\
\hline
\end{tabular}

$P$ value was based on the incremental value compared with the previous model which was adjusted for age, sex, a history of diabetes, hypertension, and cardiovascular disease, systolic and diastolic blood pressure, body mass index, albumin, fasting glucose, triglyceride, total cholesterol, hemoglobin, eGFR, total calcium, phosphorous, CaXP product, uric acid, urine proteinto-creatinine ratio, ACEI and/or ARB use, and acute kidney injury episode.

investigations to compare the significance of employing the different approaches for estimating eGFR variability.

In conclusion, our results demonstrate that the greater eGFR variability by AUC\% is associated with the higher risk of progression to RRT. The formula of estimating eGFR variability by AUC method may provide a significant predictive value and guide the treatment strategies and choices in CKD patients.

\section{Conflict of Interests}

The authors declare no conflict of interests in this study.

\section{References}

[1] A. S. Go, G. M. Chertow, D. Fan, C. E. McCulloch, and C.-Y. Hsu, "Chronic kidney disease and the risks of death, cardiovascular events, and hospitalization," New England Journal of Medicine, vol. 351, no. 13, pp. 1296-1305, 2004.

[2] M. Tonelli, N. Wiebe, B. Culleton et al., "Chronic kidney disease and mortality risk: a systematic review," Journal of the American Society of Nephrology, vol. 17, no. 7, pp. 2034-2047, 2006.

[3] Z. Al-Aly, S. Balasubramanian, J. R. McDonald, J. F. Scherrer, and A. M. O'Hare, "Greater variability in kidney function is associated with an increased risk of death," Kidney International, vol. 82, no. 11, pp. 1208-1214, 2012.

[4] W. E. Mitch, M. Walser, G. A. Buffington, and J. Lemann Jr., "A simple method of estimating progression of chronic renal failure," The Lancet, vol. 2, no. 7999, pp. 1326-1328, 1976.

[5] K. Matsushita, E. Selvin, L. D. Bash, N. Franceschini, B. C. Astor, and J. Coresh, "Change in estimated GFR associates with coronary heart disease and mortality," Journal of the American Society of Nephrology, vol. 20, no. 12, pp. 2617-2624, 2009.

[6] R. M. Perkins, I. D. Bucaloiu, H. L. Kirchner, N. Ashouian, J. E. Hartle, and T. Yahya, "GFR decline and mortality risk among patients with chronic kidney disease," Clinical Journal of the American Society of Nephrology, vol. 6, no. 8, pp. 1879-1886, 2011.

[7] L. A. Stevens, J. Coresh, C. H. Schmid et al., "Estimating GFR using serum cystatin $\mathrm{C}$ alone and in combination with serum creatinine: a pooled analysis of 3,418 individuals with CKD," American Journal of Kidney Diseases, vol. 51, no. 3, pp. 395-406, 2008.

[8] M. G. Shlipak, R. Katz, B. Kestenbaum et al., "Rapid decline of kidney function increases cardiovascular risk in the elderly," Journal of the American Society of Nephrology, vol. 20, no. 12, pp. 2625-2630, 2009.

[9] D. E. Rifkin, M. G. Shlipak, R. Katz et al., "Rapid kidney function decline and mortality risk in older adults," Archives of Internal Medicine, vol. 168, no. 20, pp. 2212-2218, 2008.

[10] Z. Al-Aly, A. Zeringue, J. Fu et al., "Rate of kidney function decline associates with mortality," Journal of the American Society of Nephrology, vol. 21, no. 11, pp. 1961-1969, 2010.

[11] T. C. Neylan, A. Brunet, N. Pole et al., "PTSD symptoms predict waking salivary cortisol levels in police officers," Psychoneuroendocrinology, vol. 30, no. 4, pp. 373-381, 2005.

[12] Z. Bhagwagar, S. Hafizi, and P. J. Cowen, "Increased salivary cortisol after waking in depression," Psychopharmacology, vol. 182, no. 1, pp. 54-57, 2005.

[13] T. W. Buchanan, S. Kern, J. S. Allen, D. Tranel, and C. Kirschbaum, "Circadian regulation of cortisol after hippocampal damage in humans," Biological Psychiatry, vol. 56, no. 9, pp. 651656, 2004.

[14] S. E. Watamura, B. Donzella, D. A. Kertes, and M. R. Gunnar, "Developmental changes in baseline cortisol activity in early childhood: relations with napping and effortful control," Developmental Psychobiology, vol. 45, no. 3, pp. 125-133, 2004.

[15] A. S. Levey, J. Coresh, K. Bolton et al., "K/DOQI clinical practice guidelines for chronic kidney disease: evaluation, classification, and stratification," American Journal of Kidney Diseases, vol. 39, no. 2, pp. S1-S266, 2002.

[16] A. S. Levey, J. P. Bosch, J. B. Lewis, T. Greene, N. Rogers, and D. Roth, "A more accurate method to estimate glomerular filtration rate from serum creatinine: a new prediction equation," Annals of Internal Medicine, vol. 130, no. 6, pp. 461-470, 1999.

[17] S. Vickery, P. E. Stevens, R. N. Dalton, F. van Lente, and E. J. Lamb, "Does the ID-MS traceable MDRD equation work and is it suitable for use with compensated Jaffé and enzymatic creatinine assays?" Nephrology Dialysis Transplantation, vol. 21, no. 9, pp. 2439-2445, 2006.

[18] D. B. Fekedulegn, M. E. Andrew, C. M. Burchfiel et al., "Area under the curve and other summary indicators of repeated waking cortisol measurements," Psychosomatic Medicine, vol. 69, no. 7, pp. 651-659, 2007.

[19] J. C. Pruessner, C. Kirschbaum, G. Meinlschmid, and D. H. Hellhammer, "Two formulas for computation of the area under the curve represent measures of total hormone concentration versus time-dependent change," Psychoneuroendocrinology, vol. 28, no. 7, pp. 916-931, 2003.

[20] A. M. O’Hare, A. Batten, N. R. Burrows et al., "Trajectories of kidney function decline in the 2 years before initiation of longterm dialysis," The American Journal of Kidney Diseases, vol. 59, no. 4, pp. 513-522, 2012. 
[21] S. Rosansky, "Early dialysis initiation and renal function trajectory," Journal of Internal Medicine, vol. 269, no. 3, pp. 275-277, 2011.

[22] F. E. M. Murtagh, E. Murphy, and N. S. Sheerin, "Illness trajectories: an important concept in the management of kidney failure," Nephrology Dialysis Transplantation, vol. 23, no. 12, pp. 3746-3748, 2008.

[23] R. M. Perkins, H. L. Kirchner, J. E. Hartle, and I. D. Bucaloiu, "Estimated glomerular filtration rate variability and risk of endstage renal disease among patients with stage 3 chronic kidney disease," Clinical Nephrology, vol. 80, no. 4, pp. 256-262, 2013. 


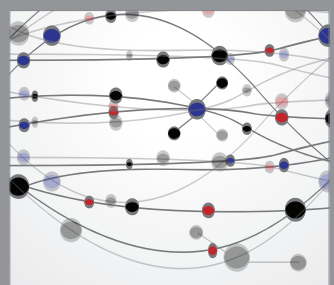

The Scientific World Journal
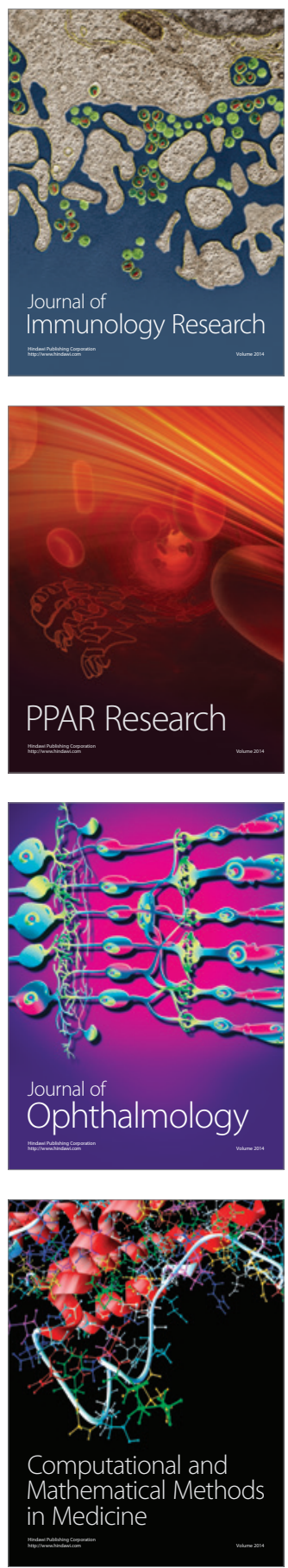

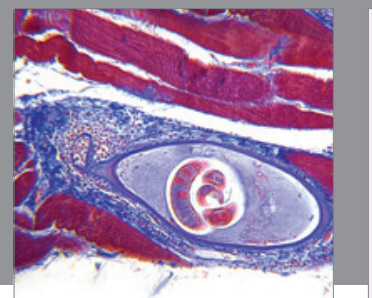

Gastroenterology

Research and Practice
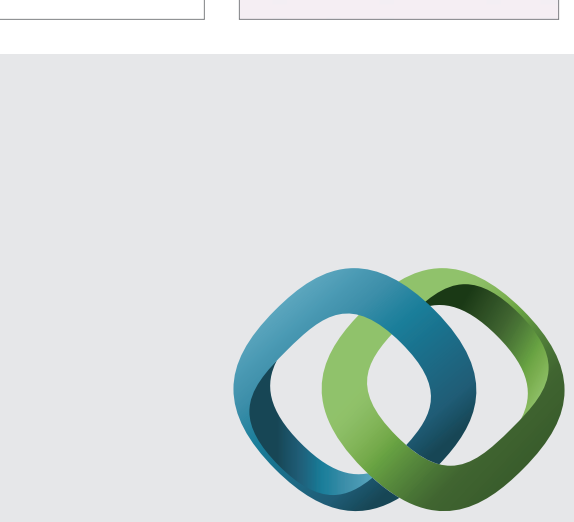

\section{Hindawi}

Submit your manuscripts at

http://www.hindawi.com
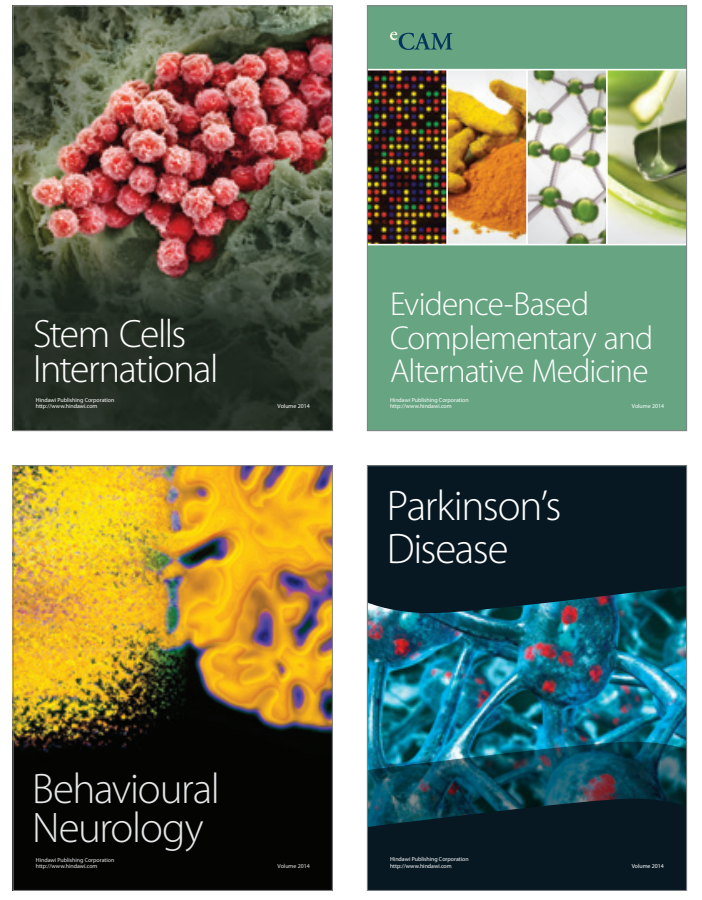
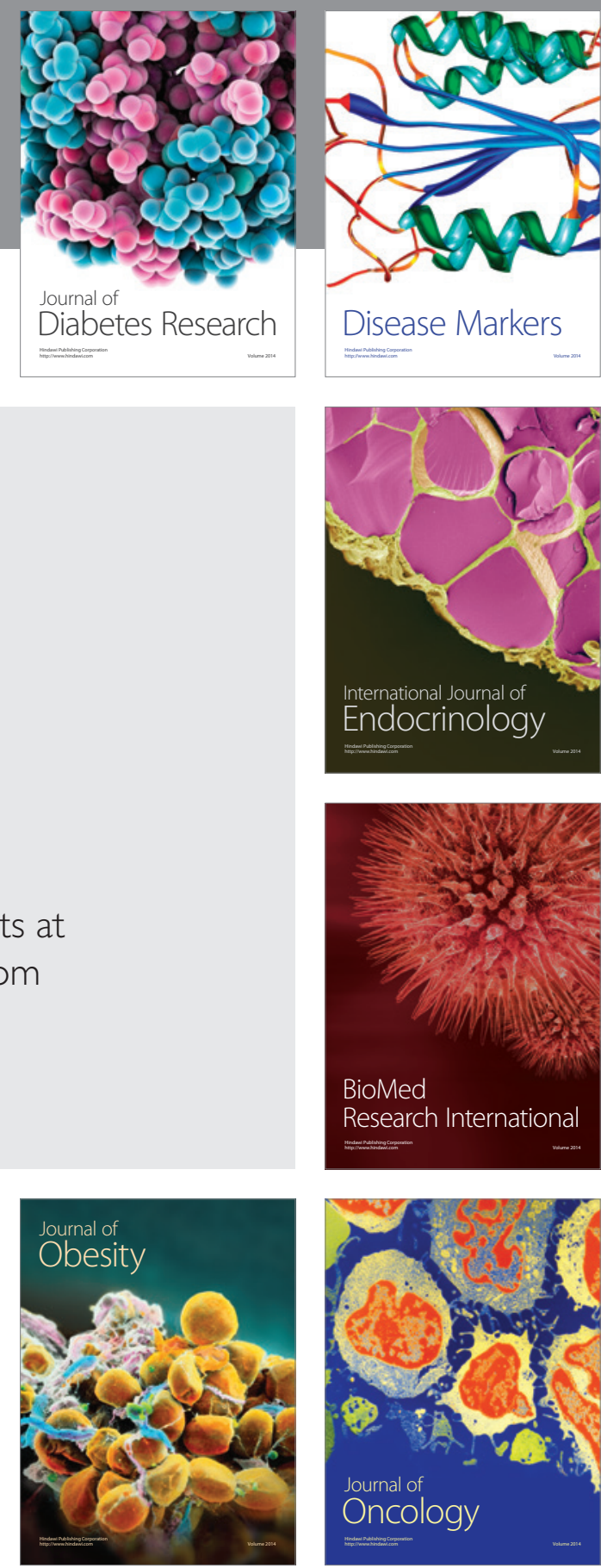

Disease Markers
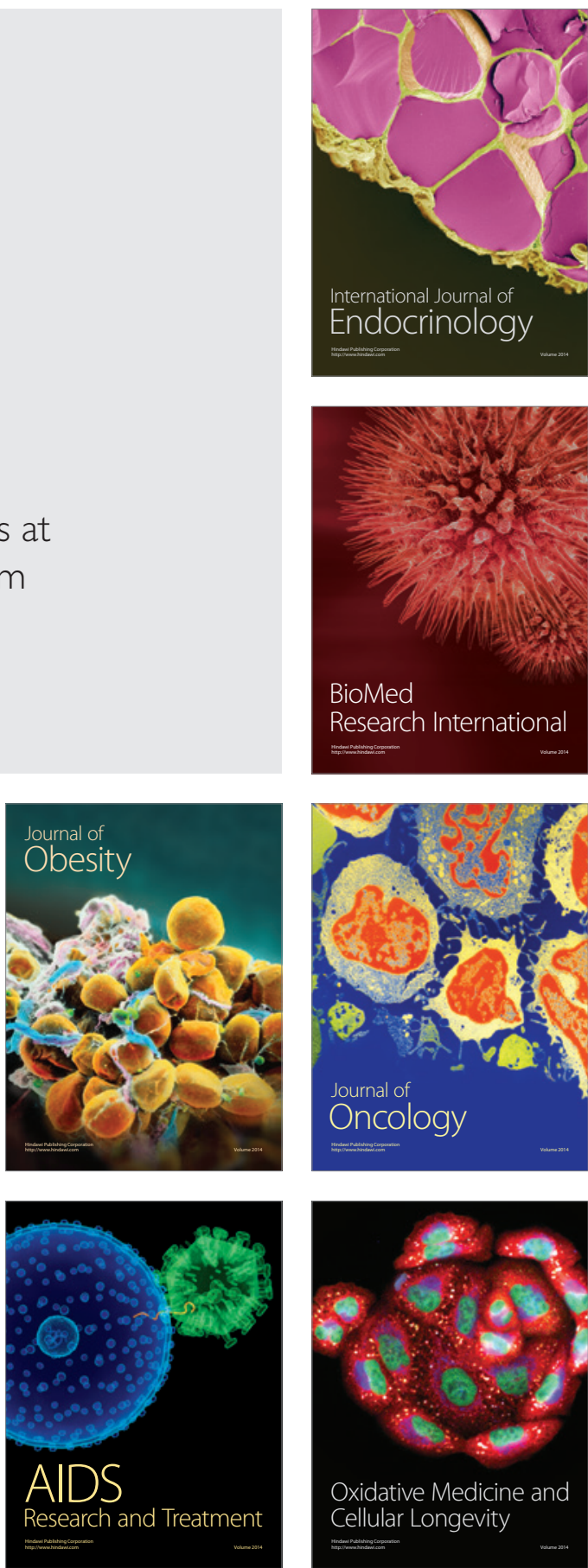